\title{
Leading safely: 'ridiculous' lessons from oil and other industries
}

\author{
Phil Hadridge
}

J R Soc Med 2005;98:539-541

'Becoming much safer is not yet a top priority for clinical leaders, executives and Boards. Other, apparently more pressing issues of organisational finance and survival occupy their attention.'

Don Berwick, to USA Senate Committee, 2003

I recently attended a meeting at the head office of one of the world's major oil companies. Having identified myself, the first thing the receptionist did was to run through the emergency procedures in the building and give me a leaflet reinforcing these instructions. In the lift the person I was visiting described the latest safety initiative within the company aimed at promoting safe travel in private and hire cars, now the major source of death and disability of staff at work.

I believe this brief insight into the central position of conversations about safety in the office environment of a large multi-national company offers a number of important lessons to the health sector - an industry where, as Berwick notes, we are struggling to put patient safety at the heart of our business.

\section{FIRST, THE IMPORTANCE OF A MOTIVATING MOMENT AND NARRATIVE}

In the early hours of 6 July 1988 on the Piper Alpha platform in the North Sea there was an explosion so sudden and massive that conventional evacuation was impossible. 167 people died. The cause was traced to flaws in design and standard operating procedures.

When talking to colleagues in the oil industry the Piper Alpha tragedy always figures in accounts of why safety is now the top issue for them. This is a story that has been told and retold. It has become a defining moment in the oil industry. It has motivated many in the most senior levels in the oil industry to see low levels of safety as unacceptable and to think in profound ways about what they can personally do to improve the safety culture.

In healthcare, what are our Piper Alphas? In the UK, The Bristol Inquiry and the Shipman Review have had a

Organizational Development Facilitator, NHS Institute for Innovation and Improvement, University of Warwick Campus, Coventry CV4 7AZ, UK

E-mail: Philip.Hadridge@dh.gsi.gov.uk profound impact, but are probably too focused on malevolent or incompetent individuals. Don Berwick has been promoting a few stories of individual family loss as (unfortunately common) examples of systemic patterns and a call to action. The public shock about MRSA in the UK may prove to be a defining moment.

\section{LEARNING FROM OTHERS- PART OF A MOVEMENT IN BUSINESS}

Second, this fundamental concern with safety is happening in many business settings. It is part of a movement where industries with haphazard approaches to the safety of staff, customers and communities are becoming 'zero tolerant' of unmanaged risks. This passionate interest in health and safety amongst senior leaders is seen in many previously high hazard industries such as oil, chemicals and air travel.

What we see is benchmarking and learning from each other: the oil industry from the chemical industry; one oil company from another-Shell from DuPont, BP with Shell. Traditional barriers are being put aside to speed to building of businesses founded on a fundamental concern for safety, for staff and customers.

\section{LESSONS FOR SHIFTING CULTURE}

Third, it tells us something about how culture changes. The lesson is that what we, and especially senior leaders, say and do is critical more than written procedures. This is important for any serious desire to develop a culture of quality in the NHS. The focus and nature of our conversations is critical.

Following Piper Alpha and other highly publicized incidents in the oil sector in the late 1980s the conversations and actions started to change. The legacy of this is seen in the safer behaviours in these operating environments.

\section{THE ROLE OF SENIOR LEADERS IN CHANGING FRONTLINE PRACTICE}

Fourth, the role of all, and especially the most senior leaders, is crucial in promoting safer practice. In the oil industry, for example, there is now a fundamental belief that until top managers behave differently about safety the frontline staff on oil rigs, in manufacturing plants or at retail outlets will not have the all the necessary incentive and 
examples to follow and a fundamental system shift will not occur. There is no doubt that what happens in remote office environments matters to practice on a distant front-line station.

Box 1 Examples of behavioural interventions by senior leaders to promote a safety culture-promoting workplace safety to signal a wider change

- When arriving at a meeting room, the most senior person checks the fire escapes are unlocked and unblocked and encourages colleagues to place bags out of the way

- The most senior person opens the meeting with a reminder of the safety procedures (e.g. any fire tests planned, congregation points, nearest phone, fire extinguisher and alarm points)

- Forbid the use of mobile phones when driving, and make allowances for this, for example in expectations of work productivity

- Encouraging staff staying in hotels to familiarise themselves with the emergency escapes and hotel safety information before retiring

- Backup junior colleagues challenging more senior staff on these issues.

If we take this example, it seems that we will be unsuccessful in our attempt to promote patient safety until senior colleagues model behaviours that put safety first. The good news is that there are simple things that can be done by all clinical and managerial leaders (Box 1). This list seems a bit odd to many at first sight; however, workplace safety is the domain that office-based senior leaders can directly influence. It is their sphere of control.

Some of the other interventions that are used to set a cultural tone for safety within the oil industry include:

- promoting safe stair walking techniques, such as holding the handrail

- providing lids for the safe carrying of coffee

- correcting colleagues tipping back on their chairs

- encouraging colleagues to refuse any form of road transport that does not provide seat belts

- regular safety tours, meetings and reviews led by senior staff.

While these actions may seem extreme in the standard office environment, they are seen as normal in oil and chemical work places: they are believed to create a context where safety is seen as important and everyone's business. A new emphasis on these sorts of measures happened in the years following Piper Alpha.

I am reminded of the words of one well-known health commentator:

'Culture change seems to be the most elusive and the most vital [ingredient in developing high performing health care organisations]_it's unpredictable and uncertain. You can't just say culture is going to change. You can't plan it as a stand alone programme - it changes through how leaders set strategy, recruit and socialise staff, structure the organisation, change technical processes. But also in less tangible ways... through purposeful conversation, the questions they ask, the stories they tell and the examples they set.'

Nigel Edwards, NHS Confederation Conference Keynote, 2002

The case of BP, Shell and other oil companies (as well as chemical, airline and other industries) brings this claim to life-they provide a relevant story from an environment the health service rarely connects with or learns from.

\section{REACTIONS AND EVIDENCE}

I have been relaying this experience to senior healthcare leaders for 3 or 4 years now. The response is often bewilderment or scepticism. I am regularly greeted with a smirk and a 'you must be joking' when I encourage someone to start an important meeting or conference with the sort of personal commitment to safety outlined above. Yet, in healthcare, I believe we need to go further, to include hand washing by senior managerial staff when visiting clinical environments, for example. However, I think it will be harder for us as our most pressing safety concerns are for our customers more than our staff.

Alternatively, I am frequently asked 'well, what is the numeric evidence this works?'. The response when asking colleagues in the oil industry and chemical industries is interesting. There is a certain belief that post Piper Alpha leaders had to do something as a personal example and that this has been profoundly enabling and reinforcing of other initiatives. In a leading chemical company they believe that this has resulted in their accident ratios being one tenth of other companies with less of a focus on safety.

So, what have we got to lose by using some of these lessons? What would happen if we acted as if personal change in the behaviours of senior leaders was a prerequisite for a system based on patient safety? Surely, the worst that can happen is to be seen as a HSE geek and fusspot. Why is this such a fear?

\section{RIDICULOUS LESSONS?}

I have been sharing this article for many months now, particularly with senior health service leaders sponsoring offsite meetings for their staff or stakeholders. The typical response to my invite for them to start the meeting with a detailed safety briefing demonstrates my stand is seen as 'faintly ridiculous'. Many of those who are personally leading work promoting patient safety find the arguments and examples above hard to stomach and are resistant of putting them into practice. 
However, there is incredulity from colleagues in oil and chemical industries when I describe the reticence of many healthcare leaders to pay attention to safety issues in meeting and conference environments. 'That's ridiculous' said a senior colleagues from a chemical company that has led the way in promoting safety for over 150 years.

My various hypotheses for this contrast in reaction is:

1 the argument I am making for drawing lessons from work on staff/system safety in other industries for patient safety in health care is wrong

2 I am arguing the case badly!

3 The health service is insufficiently curious and not fully committed to transforming our patient safety record.

On balance, following my checking with many colleagues about the logic of the case I am making and the way I am presenting it, I am drawn to hypothesis 3: this is a signifier of a service that is not about to make a profound and lasting breakthrough on patient safety.

\section{IN CLOSING}

The NHS is operating in a context where clinical staff are increasingly urged to pay attention to patient safety and where patients and the media are increasingly concerned about the potential for adverse events in health settings. The recently launched US Saving ' $110 \mathrm{k}$ lives' campaign by the Institute of Health Care Improvement (with the slogan 'Some is not a number Soon is not a time') is a sign of increasing commitment to promoting safety.

In the UK and elsewhere, leaders want to know how to act and are acting as role models for others. This article offers hope and some pointers for action by senior staff beyond important routines such safety walkabouts, promoting risk management and incident reporting mechanisms.

What leaders say and do sets the climate in organizations. There is a saying, 'first things are fateful' ... what we pay attention to has a profound impact. I believe this is true for patient safety. The spheres where managers have a direct influence are in the office, conference and meeting environments. It is here that what may seem like trivial actions, such as paying attention to safety announcements, appears crucial — and offers a clear role for office-based leaders in setting an example for patient safety. Modelling the behaviours they want to see is an important lesson - if we want staff to think safety on all occasions then leaders must do likewise. In learning from the other sectors we see that the most senior staff must develop a passion for championing health and safety in the workplace - quite a challenge in contexts where this is traditionally seen as something for junior staff alone.

If the choice for leaders is to exhort clinicians, encourage systems or model a passion for safety in their own behaviour then the lesson from the oil industry is that the latter needs more attention. This is a message for chief executives, senior officials and politicians - and indeed anyone who runs a meeting or leads a team.

I believe this is a missing ingredient in the current patient safety agenda. This is another chance for senior leaders to do something: in the first rather than third person. To 'walk the talk' themselves.

Acknowledgments Phil Hadridge would like to thank Jim Easton, Stephen Ramsden, Hugh Rogers, Stephen Thornton, David Fillingham, Diane Parker, Niall Dickson, Dick Stockford, Julia Taylor and colleagues in BP, Shell and DuPont for help and inspiration in shaping this article.

Note: Phil Hadridge is happy to host an informal discussion about the issues raised in this piece, illustrated with a number of video clips and other materials. Please e-mail philip.hadridge@dh.gsi.gov.uk if you are interested in taking part. 